\title{
Relationships between respiratory muscle strength, chest wall expansion, and functional capacity in healthy nonsmokers
}

\author{
Tadsawiya Padkao ${ }^{1,2}$, Orachorn Boonla ${ }^{1,2, *}$ \\ ${ }^{1}$ Faculty of Allied Health Sciences, Burapha University, Chonburi, Thailand \\ 2Exercise and Nutrition Sciences and Innovation Research Unit, Burapha University, Chonburi, Thailand
}

Chest wall expansion determines lung volume and functional capacity. Factors determining chest wall expansion include respiratory muscle strength. Accordingly, there may be association among respiratory muscle strength, chest wall expansion, and functional capacity. Thusly, this cross-sectional study identified the relationship between respiratory muscle strength, chest wall expansion, and functional capacity. Seventy-six healthy male and female participants aged between 20 to 59 years exhibiting a body mass index of 18.5 to $22.9 \mathrm{~kg} / \mathrm{m}^{2}$ were enrolled. Inspiratory and expiratory muscle strength were evaluated via maximum inspiratory pressure (MIP) and maximum expiratory pressure (MEP), respectively. Chest wall expansion was measured through thoracic circumferences at three different levels; upper, middle, and lower levels with diaphragmatic movement also measured utilizing the circumference of the lower edge of the 10th rib. Functional capacity was evaluated through distance obtained from a 6-min walk test. Results showed that both MIP and MEP were significantly correlated with chest wall expansion at the middle $(P=0.005$ and $P=0.006)$ and lower $(P=0.012$ and $P=0.020)$ thoracic levels as well as diaphragmatic movement (both $P<0.001)$. Moreover, both MIP and MEP were significantly related amid the 6 -min walk distance $(P=0.025$ and $P<0.001)$. In conclusion, respiratory muscle strength is positively associated with chest wall expansion, diaphragmatic movement, and functional capacity in healthy participants.

Keywords: Respiratory muscle strength, Maximum inspiratory pressure, Maximum expiratory pressure, Chest wall expansion, Functional capacity

\section{INTRODUCTION}

The degree of chest wall mobility depends on several determinants including elasticity of soft tissue structures surrounding the thorax, chest shape, and strength of the respiratory muscles (Lanza Fde et al., 2013). Chest wall expansion may be useful in clinical practice to evaluate chest wall mobility (Reddy et al., 2019). Increased chest wall expansion could be a result of increased inspiratory and expiratory muscle strength (Okrzymowska et al., 2019).

Ventilation is elevated during physical activity due to an increase in both the frequency and depth of breathing. The increase in breathing depth during training is performed by greater con- traction of the external intercostal muscle, diaphragm, and accessory muscles.

Maximum inspiratory pressure (MIP) and maximum expiratory pressure (MEP) are defined as indicators of inspiratory and expiratory muscle strength, respectively (Schoser et al., 2017). The elevation of inspiratory muscle strength could be, in part, responsible for generating more negative pressure in the pleural cavity and increased lung volume (Lutfi, 2017). The degree of chest expansion is not only due to movement of the rib cage and the external intercostal muscle expanding the transverse dimensions, but it is also associated with contraction of the diaphragm amid expanding the vertical diameter of the thoracic cavity. Moreover, the increase
${ }^{\star}$ Corresponding author: Orachorn Boonla (D https://orcid.org/0000-0002-6629-9290 Faculty of Allied Health Sciences, Burapha University, Chonburi 2013, Thailand E-mail: orachorn@go.buu.ac.th

Received: February 8, 2020 / Accepted: February 29, 2020
This is an Open Access article distributed under the terms of the Creative Commons Attribution Non-Commercial License (https://creativecommons.org/licenses/by-nc/4.0/) which permits unrestricted non-commercial use, distribution, and reproduction in any medium, provided the original work is properly cited. 
of expiratory volume could be result to promote more inspiratory volume. Therefore, either inspiratory muscle, or expiratory muscle weaknesses impact on lung volume.

Respiratory muscle strength is a proven predictor of the longterm outcomes concerning neuromuscular disease (Schoser et al., 2017). Furthermore, MIP and MEP may be more sensitive in detecting early respiratory muscle dysfunction compared with spirometry (Chiang et al., 2018; Schoser et al., 2017). Fatigue or weakness of the respiratory muscles has been reported as significantly related to exercise limitation in healthy individuals, athletes, and patients with heart failure (Amann, 2012; Janssens et al., 2013; Lin et al., 2012; Okrzymowska et al., 2019). Exercise capacity or functional capacity expresses an individual's ability to perform submaximal activities that require the integrated efforts and health of the pulmonary, cardiovascular, and skeletal muscle systems (Arena et al., 2007). A clinical trial in patients with chronic obstructive pulmonary disease demonstrated that pulmonary function was positively correlated with reduction of chest wall expansion (Kaneko et al., 2016). Moreover, a cross-sectional study in individuals with sickle cell disease revealed significant correlations between pulmonary function (maximum voluntary ventilation) and MEP, as well as between peripheral muscle strength (handgrip strength), MIP, and the 6MWD (Ohara et al., 2014).

Considering that respiratory muscle strength may be associated with chest wall expansion and functional capacity, it may be assumed that these variables correlate with each other. Hence, this study aimed to identify the correlations between respiratory muscle strength, chest wall expansion, and functional capacity. Findings from this study may be applied to pulmonary and cardiac rehabilitation programs, i.e., respiratory muscle training in patients with reduced chest wall expansion and lung volume caused by respiratory muscle weakness or thoracic surgery in order to improve recovery and activities of daily living.

\section{MATERIALS AND METHODS}

\section{Study design and participants}

This cross-sectional study was carried out in Mueang Chonburi District, Chonburi Province, Thailand between December 2016 and March 2017. A total of 76 participants (34 males and $42 \mathrm{fe}-$ males) aged 20 to 59 years were enrolled. During screening, participants were interviewed by a researcher on health and smoking status. Together, they were examined for height, body mass, body mass index (BMI), blood pressure (BP), and pulmonary function. Inclusion criteria included: (a) aged 20-59 years; (b) BMI 18.50-
$22.90 \mathrm{~kg} / \mathrm{m}^{2}$; (c) a nonsmoker; (d) normal pulmonary function test. Exclusion criteria included: (a) had history of respiratory disease (i.e., asthma, bronchitis, emphysema), cardiovascular disease (i.e., coronary artery disease, myocardial infarction, hypertension), and neuromuscular disease (i.e., pain, weakness); and (b) abnormal chest shape (i.e., barrel chest, kyphosis). All participants were informed of the study information including protocols, details, risks, and their role-both in writing and verbally prior to screening and signing of the consent form. All procedures in this study were approved by the Institutional Ethics Committee of the Faculty of Allied Health Sciences, Burapha University (approval number: 04/2560 and 05/2560), and in accordance with the ethical standards of the Declaration of Helsinki.

\section{Sample size}

Sample size was calculated using the Yamane formula. Population statistics reported in December 2015 showed that there were 916,476 people aged 20-59 years dwelling in Mueang Chonburi District, Chonburi Province, Thailand. By substituting all numbers into the formula, i.e., $\mathrm{N}=$ population size $(916,476), \mathrm{e}=$ margin of error (0.12), $\mathrm{n}$ (corrected sample size) is then equal to at least 70 persons.

\section{Study protocols}

During the week following screening, participants who were selected based on the inclusion/exclusion criteria and provision of informed consent, partook in the study. Participants were evaluated for chest wall expansion followed by respiratory muscle strength (inspiratory and expiratory muscle strength), and functional capacity. All participants were evaluated under similar conditions, namely in the morning and after a meal. Test sequence was considered based on the extent of effort ordered from less to more. Each test was conducted by the same researcher based on best intrapersonal reliability.

\section{Measurement of pulmonary function}

All participants were screened for pulmonary function using a calibrated CareFusion MicroLab spirometer (CareFusion MicroLab spirometer, CareFusion UK 232 Ltd., Kent, UK). Pulmonary function parameters including forced vital capacity (FVC) and forced expiratory volume in $1 \sec \left(\mathrm{FEV}_{1}\right)$ were measured and interpreted based on the reference values obtained from Thai, healthy nonsmokers (Dejsomritrutai et al., 2000). Each participant was sat in the sitting position wearing a nose-clip. The researcher then explained and demonstrated a correct maneuver. Each participant 
was asked to fully inhale through a mouthpiece, sustained for less than one second, and then instructed to force an exhalation for as long as possible through a mouthpiece. Participants were then instructed to exhale until no more air could be expelled. This measurement was repeated over the course of three maneuvers with the greatest value was then reported. Each participant was asked to perform further maneuvers if he/she had not met the criteria. However, no more than eight maneuvers were usually required (Miller et al., 2005). The spirometer was calibrated using a 1-L syringe connected to the transducer as previously described following a spirometry standardization method. A calibration check of inspiration and expiration report of less than $3 \%$ was considered passed.

\section{Measurement of respiratory muscle strength}

Inspiratory and expiratory muscle strength were measured via MIP and MEP employing a calibrated respiratory pressure meter (MicroRPM, CareFusion UK 232 Ltd., Basingstoke, UK) with a disposable cardboard mouthpiece. Participants wore a nose-clip and were seated for the test. The researcher explained and demonstrated a correct maneuver. During the MIP maneuver, participants were instructed to exhale slowly and fully, seal his/her lips firmly around the mouthpiece, and then perform a strong inhalation through the mouthpiece with maximal effort (inhaled from residual volume). During the MEP maneuver, each participant was instructed to inhale slowly, as deeply as possible, seal his/her lips firmly around the mouthpiece, and then perform a strong exhalation through the mouthpiece with maximal effort (exhaled from total lung capacity). Each maneuver was held for 2 sec with participants instructed to perform five maneuvers with 1 min rest between maneuvers (Hautmann et al., 2000). The greatest three values were subsequently averaged and reported. Calibration was achieved by attaching the device to a pressure manometer as previously described (Miller et al., 2005).

\section{Measurement of chest wall expansion}

Chest wall expansion was measured at the thoracic level using a standard measuring tape for thoracic circumference at three different levels; upper, middle, and lower levels. Upper thoracic circumference was measured at the axillary level. Middle thoracic circumference was measured at the nipple line, while lower thoracic circumference was measured at the tip of the xiphoid process. Each participant was asked to breathe out maximally and then breathe in maximally. The difference between the two scales (breathing in minus breathing out) was determined as thoracic expansion. Diaphragmatic movement was also evaluated through abdominal cir- cumference measured at the lower edge of the 10th rib. Participants were instructed to perform three maneuvers and the obtained values were subsequently averaged and reported (Adachi et al., 2015; Lanza Fde et al., 2013).

\section{Evaluation of functional capacity}

The 6-min walk test (6MWT) is a submaximal exercise test widely used for assessing functional capacity (Du et al., 2017). Briefly, the participant is instructed to walk on a pathway as far as possible, but not run, for $6 \mathrm{~min}$. In our setup, traffic cones were placed at the start and end points of a 30-m pathway.

\section{Statistical analyses}

Data were expressed as mean \pm standard deviation. Pearson correlation coefficient was used to analyze associations of respiratory muscle strength (MIP, MEP), chest wall expansion (upper, middle, and lower thoracic circumferences), diaphragmatic movement (abdominal circumference at the 10th ribs), and functional capacity (6MWD). Correlation was considered as weak if $r$ was $<0.50$, moderate if $r$ was $0.50-0.70$, and good if $r$ was $>0.70$. All analyses were performed using IBM SPSS Statistics ver. 21 (IBM Co., Chicago, IL, USA). Statistical significance was considered as if $P<0.05$.

Table 1. Physical and physiological characteristics of participants $(n=76)$

\begin{tabular}{lc}
\hline Parameter & Mean $\pm S D$ \\
\hline Age $(\mathrm{yr})$ & $31.87 \pm 11.23$ \\
Gender & \\
Male $(\mathrm{n}=34)$ & $33.18 \pm 10.11$ \\
Female $(\mathrm{n}=42)$ & $30.80 \pm 12.10$ \\
Height $(\mathrm{m})$ & $1.64 \pm 0.08$ \\
Weight $(\mathrm{kg})$ & $57.25 \pm 6.54$ \\
Body mass index $\left(\mathrm{kg} / \mathrm{m}^{2}\right)$ & $21.16 \pm 1.47$ \\
Blood pressure $(\mathrm{mmHg})$ & \\
Systolic blood pressure & $117.93 \pm 11.64$ \\
Diastolic blood pressure & $81.87 \pm 9.94$ \\
Mean arterial pressure & $93.89 \pm 9.49$ \\
Pulmonary function & \\
FVC (L) & $3.10 \pm 0.82$ \\
$\%$ predicted & $89.83 \pm 14.13$ \\
FEV 1 (L) & $2.83 \pm 0.69$ \\
$\%$ predicted & $95.70 \pm 15.66$ \\
FEV $/$ FVC $(\%)$ & $91.66 \pm 5.34$ \\
$\%$ predicted & $104.39 \pm 6.32$ \\
$6 \mathrm{MWD}(\mathrm{m})$ & $552.46 \pm 68.28$ \\
\hline
\end{tabular}

$\mathrm{SD}$, standard deviation; FVC, forced vital capacity; $\mathrm{FEV}_{1}$, force expiratory flow in 1 sec; 6MWD, 6-min walk distance. 


\section{RESULTS}

Physical and physiological characteristics of participants including age, gender, height, BMI, BP (systolic BP and diastolic BP), pulmonary function $\left(\mathrm{FEV}_{1}, \mathrm{FVC}\right.$, and $\left.\mathrm{FEV}_{1} / \mathrm{FVC}\right)$, and 6MWD are presented in Table 1. BMI, BP, and lung function values were

Table 2. Chest wall expansion and respiratory muscle strength of participants $(n=76)$

\begin{tabular}{lc}
\hline Parameter & Mean \pm SD \\
\hline Chest wall expansion $(\mathrm{cm})$ & \\
Upper & $4.87 \pm 1.24$ \\
Middle & $4.57 \pm 1.34$ \\
Lower & $4.85 \pm 1.49$ \\
Diaphragmatic movement (cm) & $5.01 \pm 1.25$ \\
MIP $\left(\mathrm{cmH}_{2} \mathrm{O}\right)$ & $86.48 \pm 25.75$ \\
MEP $\left(\mathrm{cmH}_{2} \mathrm{O}\right)$ & $96.02 \pm 25.91$ \\
\hline
\end{tabular}

SD, standard deviation; MIP, maximum inspiratory pressure; MEP, maximum expiratory pressure.
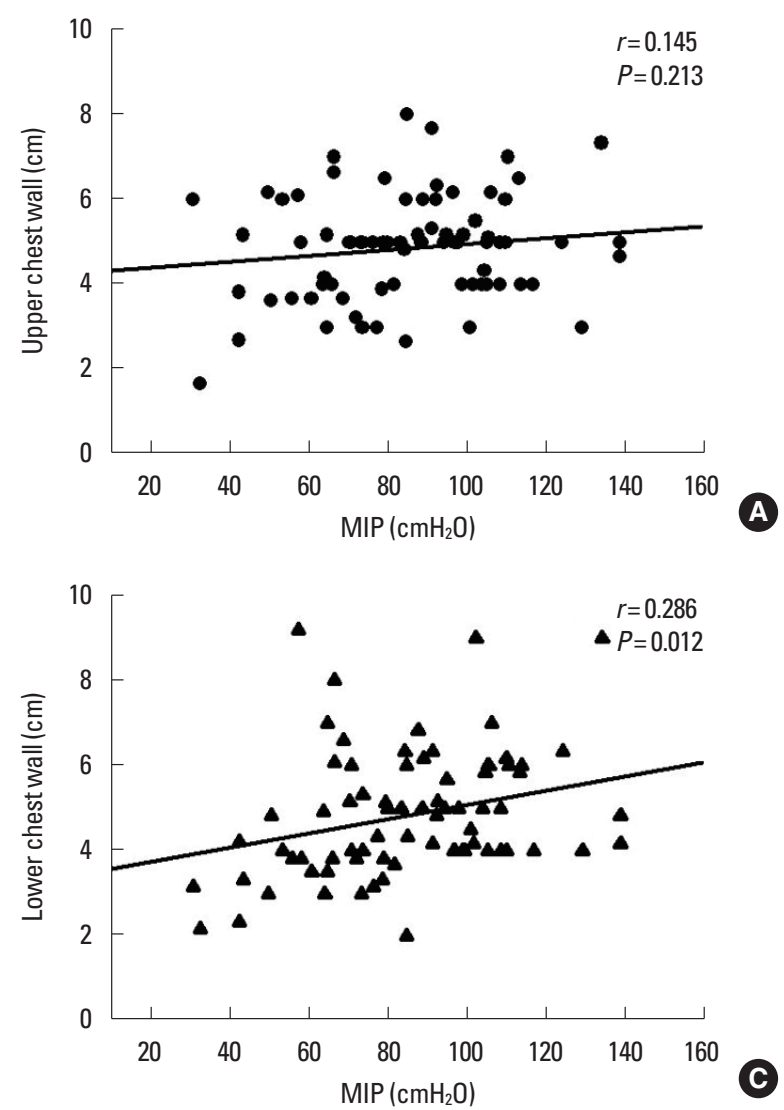

C in the normal range.

Table 2 demonstrates participants' chest wall expansion consisting of upper, middle, and lower thoracic levels, in addition to diaphragmatic movement. Inspiratory and expiratory muscle strength are indicated as MIP and MEP.

Pearson correlation analysis showed the relationship between inspiratory muscle strength and chest wall expansion. MIP was significantly positively correlated to middle $(r=0.321, P=0.005)$ and lower $(r=0.286, P=0.012)$ thoracic circumferences and diaphragmatic movement $(r=0.466, P<0.001)$. There was no relationship between inspiratory muscle strength and upper thoracic circumference (Fig. 1).

Correlation between expiratory muscle strength and chest wall expansion was observed. MEP was significantly positively correlated with middle $(r=0.315, P=0.006)$ and lower $(r=0.266, P=0.02)$ thoracic circumferences, and diaphragmatic movement $(r=0.392$, $P<0.001)$, yet was not correlated with upper thoracic circumference (Fig. 2).
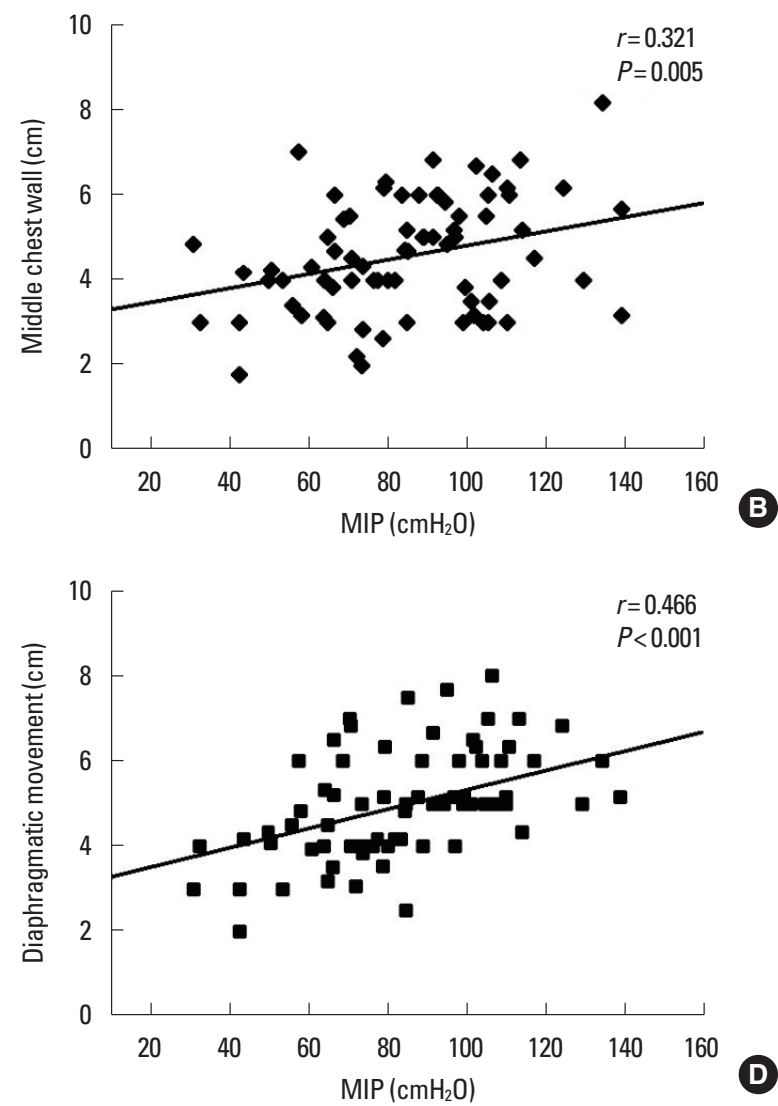

Fig. 1. Correlations between maximum inspiratory pressure (MIP) and upper (A), middle (B), lower chest wall expansion (C), and diaphragmatic movement (D). Data are expressed as mean \pm standard deviation. $n=76$. 

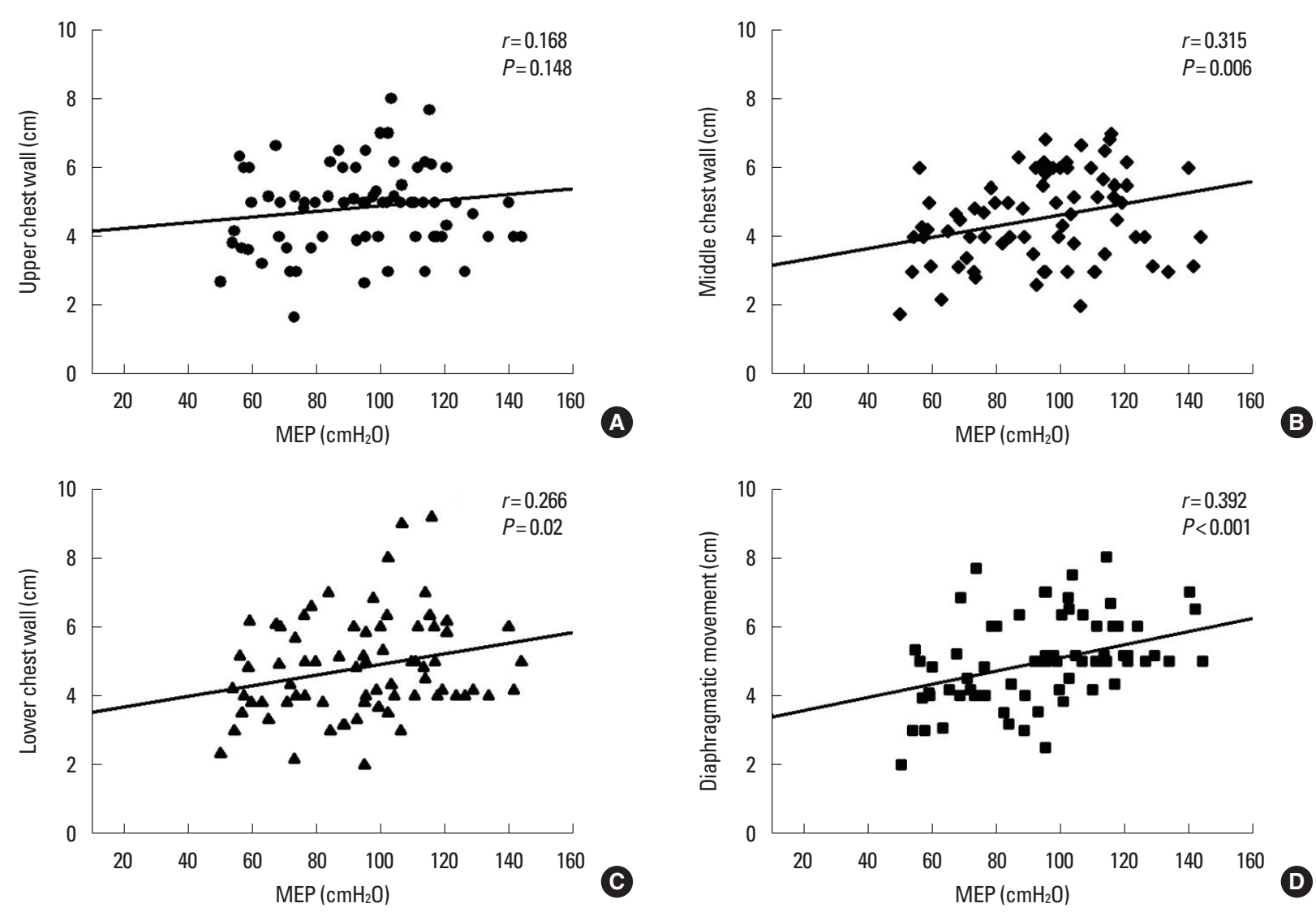

Fig. 2. Correlations between maximum expiratory pressure (MEP) and upper (A), middle (B), lower chest wall expansion (C), and diaphragmatic movement (D). Data are expressed as mean \pm standard deviation. $n=76$.
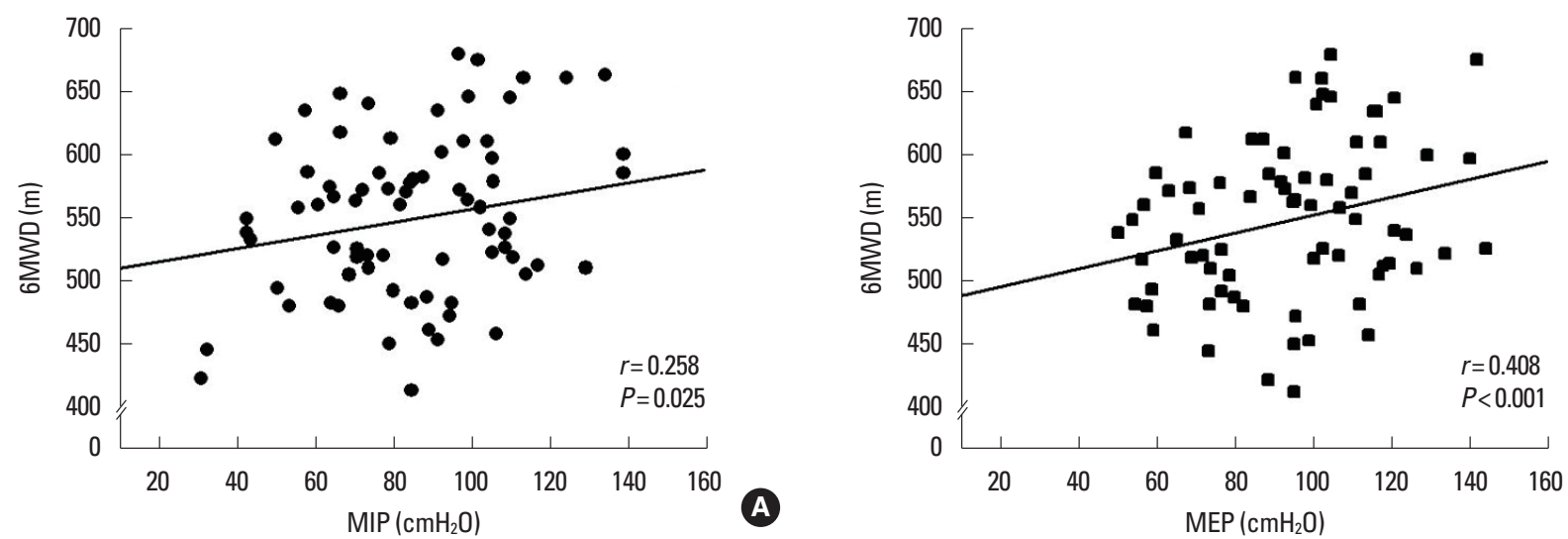

Fig. 3. Correlations between maximum inspiratory pressure (MIP) and 6-min walk distance (6MWD) (A) and maximum expiratory pressure (MEP) and 6MWD (B). Data are expressed as mean \pm standard deviation. $n=76$.

Analysis of the association between respiratory muscle strength and functional capacity is shown in Fig. 3. Consequently, the results revealed that both MIP $(r=0.025, P=0.040)$ and MEP $(r=$ $0.408, P<0.001)$ were significantly positively associated with the 6MWD.

\section{DISCUSSION}

This study identified the relationships between MIP and MEP, different levels of thoracic chest wall expansion, and 6MWD in healthy nonsmokers. The major findings demonstrated positive 
correlations between MIP and MEP, middle and lower thoracic chest wall expansion, and diaphragmatic movement. In addition, MIP and MEP were also correlated with the 6MWD.

The contraction of the inspiratory muscles; principally the diaphragm and external intercostal muscles generates force interaction amid the chest wall and increases chest wall and lung expansion. As a result, pleural pressure is decreased creating a pressure gradient that allows air flow into the lungs (Lo Mauro and Aliverti, 2016). Respiratory muscle fatigue has been reported to decrease exercise performance in healthy subjects (Witt et al., 2007; Wüthrich et al., 2013). Moreover, weakness of inspiratory muscle is correlated with low functional capacity in end-stage renal disease, chronic obstructive pulmonary disease, and heart failure patients (Du et al., 2017; Singer et al., 2011; Yamada et al., 2016). Recent results showed positive association between inspiratory muscle weakness and functional capacity as measured by distance walked during the incremental shuttle-walk test in hemodialysis patients (Figueiredo et al., 2017). Furthermore, it has been showed that an inspiratoty muscle training improved the distance walked in 6 min among patients with stable chronic heart failure and chronic obstructive pulmonary disease (Du et al., 2017; Martínez et al., 2001). In addition, after respiratory muscle training was reported to reduction of respiratory muscle fatigue and perception of exertion, as well as increased blood flow to the working muscles by reducing respiratory muscle metaboreflex, thereby enabling subjects to perform a greater walking distance (Witt et al., 2007; Wüthrich et al., 2013). Increases in MIP and MEP, indicating augmentation in inspiratory and expiratory muscle strength, have been reported to be associated with increased chest wall mobility (Lanza Fde et al., 2013; Sahin et al., 2004). Our study observed that both MIP and MEP were significantly related to middle and lower thoracic chest wall expansion as well as diaphragmatic movement. Significance values further suggest that inspiratory muscle strength may be more correlated with chest wall expansion and diaphragmatic movement than expiratory muscle strength.

However, there were no correlations between MIP and MEP, and upper thoracic chest wall expansion, which was measured at the axillary level. Moreover, our results contrast with those from previous studies which measured the association of chest wall mobility and respiratory muscle strength in healthy subjects that observed correlations between respiratory muscle strength, and both axillary and thoracic cirtometry (Lanza Fde et al., 2013). Chest wall mobility is not only associated with respiratory muscle strength but is also related to other determinants such as elasticity of the soft tissue structures surrounding the thorax, shape of the chest wall, age, gender, and BMI (Jalayondeja et al., 2014). When compared with a study by Lanza Fde et al. (2013), our participants exhibited a greater mean age (31.9 \pm 11.2 years vs. $24 \pm 3$ years), and as a result, lower respiratory muscle strength (MIP: $86.5 \pm 25.8 \mathrm{cmH}_{2} \mathrm{O}$ vs. $93.8 \pm 30.2 \mathrm{cmH}_{2} \mathrm{O}$; $\mathrm{MEP}: 96.0 \pm 25.9 \mathrm{cmH}_{2} \mathrm{O}$ vs. $103.2 \pm$ $\left.38.1 \mathrm{cmH}_{2} \mathrm{O}\right)$ and lung function $\left(\mathrm{FEV}_{1}: 2.8 \pm 0.7 \mathrm{~L}\right.$ vs. $3.7 \pm 0.7 \mathrm{~L}$; FVC: $3.1 \pm 0.8 \mathrm{~L}$ vs. $4.4 \pm 0.9 \mathrm{~L}$ ). Therefore, this evidence could partly imply that advancement in age may cause deterioration to upper chest wall expansion. Besides that, previous reports have consistently suggested that upper thoracic excursion is negatively correlated with age in healthy subjects aged $20-70$ years (Kumar, 2013).

The present study also observed positive correlations between MIP and MEP with the 6MWD. On the other hand, significance values further suggest that expiratory muscle strength may be more correlated with functional capacity than inspiratory muscle strength. Our results are in agreement with previous studies reporting the potential relationship between inspiratory muscle strength and functional capacity (Demir et al., 2018; Zeren et al., 2016). Furthermore, it has been reported that respiratory muscle weakness is associated with reduced functional capacity in hemodialysis patients (Figueiredo et al., 2017). What's more, inspiratory muscle training has been shown to produce advantageous effects in terms of increasing respiratory muscle strength and functional capacity (de Medeiros et al., 2017; Saglam et al., 2015). Increased respiratory muscle strength may enhance right ventricular function, i.e. ventricular filling and ejection fraction, whereby the latter is strongly related to peak oxygen consumption (Mancini et al., 1995). In addition, an increase in respiratory muscle strength may elevate blood flow to the working muscles via attenuation of the respiratory muscle metaboreflex as well as increase leg muscle perfusion and oxygenation, thereby enabling skeletal muscle endurance (Mancini et al., 1995; Witt et al., 2007; Wüthrich et al., 2013).

A randomized controlled trial in individuals with mild to moderate Parkinson disease suggested that increases in inspiratory muscle strength contribute to improved expiratory muscle strength, thoracic mobility, and functional capacity (Montero Ferro et al., 2019). As mentioned, it is likely that respiratory muscle strength is directly associated with chest wall expansion (Jung and Kim, 2018) and the latter directly correlates with lung volume (Ray et al., 2013). Furthermore, respiratory muscle strength also directly relates to functional capacity (Mancini et al., 1995).

Our study presents some limitations. The major restriction was that our study was conducted in a relatively small population. Hence, the results may not be thoroughly representative of larger 
populations. Conversely, we identified the associations among gathered respiratory muscle strength, chest wall expansion, diaphragmatic movement, and functional capacity. Though most presented a weak relationship, our data may additionally provide an understanding of relations between those variables.

Respiratory muscle strength, in particular inspiratory muscle strength, displays positive associations with chest wall expansion at the middle and lower thoracic levels, diaphragmatic movement, and functional capacity. Finally, these findings may be useful amid the application of physical rehabilitation: both pulmonary and cardiac.

\section{CONFLICT OF INTEREST}

No potential conflict of interest relevant to this article was reported.

\section{ACKNOWLEDGMENTS}

This study was supported by the Faculty of Allied Health Sciences, Burapha University and Exercise and Nutrition Sciences and Innovation Research Unit, Burapha University, Thailand. We would like to thank Ms. Supatta Huangdee, Ms. Adeline Siegenthaler, Ms. Kanyarat Khammak, Mr. Passawit Chongruchirotchai, Mr. Korrakot Chaikittiporn, and Ms. Piyanuch Dumrongphum for recruiting and coordinating participants.

\section{REFERENCES}

Adachi D, Yamada M, Nishiguchi S, Fukutani N, Hotta T, Tashiro Y, Morino S, Shirooka H, Nozaki Y, Hirata H, Yamaguchi M, Aoyama T. Age-related decline in chest wall mobility: a cross-sectional study among community-dwelling elderly women. J Am Osteopath Assoc 2015;115:384-389.

Amann M. Pulmonary system limitations to endurance exercise performance in humans. Exp Physiol 2012;97:311-318.

Arena R, Myers J, Williams MA, Gulati M, Kligfield P, Balady GJ, Collins E, Fletcher G; American Heart Association Committee on Exercise, Rehabilitation, and Prevention of the Council on Clinical Cardiology; American Heart Association Council on Cardiovascular Nursing. Assessment of functional capacity in clinical and research settings: a scientific statement from the American Heart Association Committee on Exercise, Rehabilitation, and Prevention of the Council on Clinical Cardiology and the Council on Cardiovascular Nursing. Circulation 2007;116:329-343
Chiang J, Mehta K, Amin R. Respiratory diagnostic tools in neuromuscular disease. Children (Basel) 2018;5:78.

de Medeiros AIC, Fuzari HKB, Rattesa C, Brandão DC, de Melo Marinho PÉ. Inspiratory muscle training improves respiratory muscle strength, functional capacity, and quality of life in patients with chronic kidney disease: a systematic review. J Physiother 2017;63:76-83.

Dejsomritrutai W, Nana A, Maranetra KN, Chuaychoo B, Maneechotesuwan K, Wongsurakiat P, Chierakul N, Charoenratanakul S, Tscheikuna J, Juengprasert W, Suthamsmai T, Naruman C. Reference spirometric values for healthy lifetime nonsmokers in Thailand. J Med Assoc Thai 2000;83:457-466.

Demir R, Zeren M, Gurses HN, Yigit Z. Relationship of respiratory muscle strength, pulmonary function, and functional capacity with quality of life in patients with atrial fibrillation. J Int Med Res 2018;46:195-203.

Du H, Wonggom P, Tongpeth J, Clark RA. Six-minute walk test for assessing physical functional capacity in chronic heart failure. Curr Heart Fail Rep 2017;14:158-166.

Figueiredo PH, Lima MM, Costa HS, Gomes RT, Neves CD, Oliveira ES, Alves FL, Rodrigues VG, Maciel EH, Balthazar CH. The role of the inspiratory muscle weakness in functional capacity in hemodialysis patients. PLoS One 2017;12:e0173159.

Hautmann H, Hefele S, Schotten K, Huber RM. Maximal inspiratory mouth pressures (PIMAX) in healthy subjects—what is the lower limit of normal? Respir Med 2000;94:689-693.

Jalayondeja W, Verner O, Jarungjitaree S, Tscheikuna J. Respiratory muscle strength explained by age and weight in female and male. J Med Assoc Thai 2014;97 Suppl 7:S16-20.

Janssens L, Brumagne S, McConnell AK, Raymaekers J, Goossens N, GayanRamirez G, Hermans G, Troosters T. The assessment of inspiratory muscle fatigue in healthy individuals: a systematic review. Respir Med 2013;107:331-346.

Jung JH, Kim NS. Changes in training posture induce changes in the chest wall movement and respiratory muscle activation during respiratory muscle training. J Exerc Rehabil 2018;14:771-777.

Kaneko H, Shiranita S, Horie J, Hayashi S. Reduced chest and abdominal wall mobility and their relationship to lung function, respiratory muscle strength, and exercise tolerance in subjects with COPD. Respir Care 2016;61:1472-1480.

Kumar SP. Physical therapy and central sensitization: are we explaining to patients with 'unexplained' pain? J Phys Ther 2013;6:41-45.

Lanza Fde C, de Camargo AA, Archija LR, Selman JP, Malaguti C, Dal Corso $S$. Chest wall mobility is related to respiratory muscle strength and lung volumes in healthy subjects. Respir Care 2013;58:2107-2112.

Lin SJ, McElfresh J, Hall B, Bloom R, Farrell K. Inspiratory muscle training in patients with heart failure: a systematic review. Cardiopulm Phys 
Ther J 2012;23:29-36.

Lo Mauro A, Aliverti A. Physiology of respiratory disturbances in muscular dystrophies. Breathe (Sheff) 2016;12:318-327.

Lutfi MF. The physiological basis and clinical significance of lung volume measurements. Multidiscip Respir Med 2017;12:3.

Mancini DM, Henson D, La Manca J, Donchez L, Levine S. Benefit of selective respiratory muscle training on exercise capacity in patients with chronic congestive heart failure. Circulation 1995;91:320-329.

Martínez A, Lisboa C, Jalil J, Muñoz V, Díaz O, Casanegra P, Corbalán R, Vásquez AM, Leiva A. Selective training of respiratory muscles in patients with chronic heart failure. Rev Med Chil 2001;129:133-139.

Miller MR, Hankinson J, Brusasco V, Burgos F, Casaburi R, Coates A, Crapo R, Enright P, van der Grinten CP, Gustafsson P, Jensen R, Johnson DC, MacIntyre N, McKay R, Navajas D, Pedersen OF, Pellegrino R, Viegi G, Wanger J; ATS/ERS Task Force. Standardisation of spirometry. Eur Respir J 2005;26:319-338.

Montero Ferro A, P Basso-Vanelli R, Moreira Mello RL, Sanches GarciaAraujo A, Gonçalves Mendes R, Costa D, Gianlorenço AC. Effects of inspiratory muscle training on respiratory muscle strength, lung function, functional capacity, and cardiac autonomic function in Parkinson's disease: randomized controlled clinical trial protocol. Physiother Res Int 2019;24:e1777.

Ohara DG, Ruas G, Walsh IA, Castro SS, Jamami M. Lung function and six-minute walk test performance in individuals with sickle cell disease. Braz J Phys Ther 2014;18:79-87.

Okrzymowska P, Kurzaj M, Seidel W, Rożek-Piechura K. Eight weeks of inspiratory muscle training improves pulmonary function in disabled swimmers-a randomized trial. Int J Environ Res Public Health 2019; 16:1747.

Ray AD, Udhoji S, Mashtare TL, Fisher NM. A combined inspiratory and expiratory muscle training program improves respiratory muscle strength and fatigue in multiple sclerosis. Arch Phys Med Rehabil 2013;94:1964-1970.

Reddy RS, Alahmari KA, Silvian PS, Ahmad IA, Kakarparthi VN, Renga- ramanujam K. Reliability of chest wall mobility and its correlation with lung functions in healthy nonsmokers, healthy smokers, and patients with COPD. Can Respir J 2019;2019:5175949.

Saglam M, Arikan H, Vardar-Yagli N, Calik-Kutukcu E, Inal-Ince D, Savci S, Akdogan A, Yokusoglu M, Kaya EB, Tokgozoglu L. Inspiratory muscle training in pulmonary arterial hypertension. J Cardiopulm Rehabil Prev 2015;35:198-206.

Sahin G, Calikoğlu M, Ozge C, Incel N, Biçer A, Ulşubaş B, Güler H. Respiratory muscle strength but not BASFI score relates to diminished chest expansion in ankylosing spondylitis. Clin Rheumatol 2004;23: 199-202.

Schoser B, Fong E, Geberhiwot T, Hughes D, Kissel JT, Madathil SC, Orlikowski D, Polkey MI, Roberts M, Tiddens HA, Young P. Maximum inspiratory pressure as a clinically meaningful trial endpoint for neuromuscular diseases: a comprehensive review of the literature. Orphanet J Rare Dis 2017;12:52.

Singer J, Yelin EH, Katz PP, Sanchez G, Iribarren C, Eisner MD, Blanc PD. Respiratory and skeletal muscle strength in chronic obstructive pulmonary disease: impact on exercise capacity and lower extremity function. J Cardiopulm Rehabil Prev 2011;31:111-119.

Witt JD, Guenette JA, Rupert JL, McKenzie DC, Sheel AW. Inspiratory muscle training attenuates the human respiratory muscle metaboreflex. J Physiol 2007;584(Pt 3):1019-1028.

Wüthrich TU, Notter DA, Spengler CM. Effect of inspiratory muscle fatigue on exercise performance taking into account the fatigue-induced excess respiratory drive. Exp Physiol 2013;98:1705-1717.

Yamada K, Kinugasa Y, Sota T, Miyagi M, Sugihara S, Kato M, Yamamoto K. Inspiratory muscle weakness is associated with exercise intolerance in patients with heart failure with preserved ejection fraction: a preliminary study. J Card Fail 2016;22:38-47.

Zeren M, Demir R, Yigit Z, Gurses HN. Effects of inspiratory muscle training on pulmonary function, respiratory muscle strength and functional capacity in patients with atrial fibrillation: a randomized controlled trial. Clin Rehabil 2016;30:1165-1174. 